\title{
Papular-purpuric gloves and socks syndrome due to parvovirus B19: a report of two simultaneous cases in cohabitant families
}

\author{
S. Bello, R. Fanizzi, C. Bonali, L. Serafino, N. Terlizzi, G. Lapadula \\ Rheumatology Unit, Medical School, University of Bari, Italy
}

\begin{abstract}
SUMMARY
The so-called papular-purpuric gloves and socks syndrome (PPGSS) is a condition characterized by acute onset of intense erythema, edema and petechiae with a typical localization on the hands and feet, besides mucosal lesions of the oral cavity. The syndrome has a favorable and self-limited course, requiring only a symptomatic therapy. In the $50 \%$ of the cases described in literature (ninety cases in 22 years), is documented an acute infection caused by parvovirus B19 and in only two cases the onset of PPGSS is reported among different members of the same family.

The aim of the work is to describe two cases of PPGSS arisen during a short time period in two family members affected by an acute parvovirus B19 infection found by serum sampling. The peculiarity of the study was the infrequence of the syndrome and the rareness of the description of PPGSS in rheumatology. This syndrome is usually described in dermatology, but it is also interesting for the rheumatologist because it comes in differential diagnosis with various autoimmune diseases.
\end{abstract}

Key words: Parvovirus B 19, PPGSS, gloves and socks syndrome.

Reumatismo, 2013; 65 (1): 40-45

\section{INTRODUCTION}

arvovirus B19 is a small DNA virus belonging to the Parvoviridae family and it is also the only parvovirus pathogenic for humans. Parvovirus B19 infection is spread worldwide and occurs, with higher incidence, during winter and spring. The virus is transmitted through exposure to infected respiratory droplets or blood products. The incubation period varies between 4 and 28 days and the contagiousness is limited to this period.

In the general population the seropositivity is $30-60 \%$ among adults and greater than $80 \%$ in the elderly. Most of parvovirus B19 infection are asymptomatic; but the most common clinical presentations include cutaneous manifestations (e.g. megaloeritema), hematological disorders (e.g. transient aplastic crisis), rheumatic mani- festations (e.g. non-erosive polyarthritis) and the gloves-socks syndrome, or papular-purpuric gloves and socks syndrome (PPGSS). This infection when contracted during pregnancy may cause miscarriage or fetal hydrops. The erythema infectiosum also called fifth disease or megaloeritema has a benign, self-limited course and it appears as rubella-like maculo-papular erythematous rash, localized to the cheeks, the trunk and the extremities. The joint involvement, most frequent in adulthood, is characterized by symptoms ranging from diffuse arthralgias with morning stiffness, to a real arthritis; in adults it occurs with symmetric polyarthritis, while in children prevails the oligoarticular involvement of lower limbs; the symptoms resolve themselves within 2-4 weeks. Patients affected by impaired bone marrow function may show a transient aplastic crisis, a condi- 
tion characterized by erythroid hypoplasia with significant reductions in hemoglobin and reticulocytopenia until the complete disappearance of circulating reticulocytes; lymphocytosis, eosinophilia, neutropenia and thrombocytopenia are often observed. The infection contracted during pregnancy in the $33 \%$ of the cases may determine the transplacental passage of the virus with the development of fetal hydrops or miscarriage (1).

PPGSS is a rare manifestation of parvovirus B19 infection characterized by acute onset and a favorable, self-limited course, marked by erythema, purple on hands and feet, fever and oral lesions. The cutaneous manifestation, often painful and itchy, is localized on hands and feet with a typical gloves and socks distribution, followed by the appearance of petechiae and rarely, blisters or bubbles. These lesions persist few days and may develop into areas of scaling, until a restitutio ad integrum (2, $3)$. Less frequently they may involve other areas e.g. the root of the limbs, trunk and face $(4,5)$. Further lesions of the oral cavity are common and are represented by erythema, petechiae and erosions of the palate besides by edema and erythema of the lip $(6,7)$. The systemic involvement, which may precede or develop together with the cutaneous manifestations, includes flu-like symptoms (e.g, fever, fatigue, lymphadenopathy, myalgia, arthralgia) and gastrointestinal ones (e.g. diarrhea and abdominal pain).

Although 50\% of the cases of PPGSS have been reported to be caused by parvovirus $\mathrm{B} 19$, it is also described the relationship with other etiological agents: viruses (cytomegalovirus, measles, rubella, Hepatitis $\mathrm{B}$ virus, coxsackie virus B6, herpes virus 6 and 7), arcanobacterium haemoliticum, mycoplasma pneumonia and therapy with trimethoprim sulfametoxazole (8-14).

Since the first description of 5 cases in 1990 in Switzerland by Harms et al. (2), about 90 cases of PPGSS have been described in literature (74 items in PubMed for papular-purpuric gloves and socks syndrome) and only two publications report the occurrence of PPGSS among several members of the same family $(15,16)$. The motivation of this study came from the rarity of the syndrome itself, particularly because diagnosed in two members of the same family.

\section{CASE REPORT}

In March 2011 a 30 year-old woman, Caucasian, physical therapist, was admitted as outpatient to the Rheumatology Unit of Policlinico of Bari on the advice of a dermatologist; she refers a 4 days history of fever $\left(39.1^{\circ} \mathrm{C}\right)$, painful aphthous lesions of the mouth, abdominal pain with episodic diarrhea, arthralgia involving the wrists, the knees and the ankles and hyperemicpapular rash initially localized to hands and feet (Fig. 1 and 2) and later extended to the root of the limbs and trunk. Since the onset of the symptoms the patient lived in the same house with her husband, the mother, the father and a two years old son; the baby is not yet attending kindergarten and remained asymptomatic before and during his mother's illness. In the same period there was no news of exceptional spread of infectious diseases in the areas frequented by the patient and her family.

We found after examination an intense erythema of the four extremities with a clear gloves-socks distribution and petechial lesions. Large and irregular ulcers of the mucosa of the palate and lip were also present. The diagnostic investigations resulted normal for: blood counts, liver, kidney and thyroid function tests, serum electrolytes, muscle enzymes, C3 fraction of complement, tumor markers and some of the infectious tests (antistreptolysin title, $\mathrm{B}$ and $\mathrm{C}$ hepatitis virus markers); while resulted altered the acute phase reactants (ESR $26 \mathrm{~mm}$, CRP $33.4 \mathrm{mg} / \mathrm{L}$, alpha2 globulin $13.2 \%)$, ferritin $(526 \mathrm{ng} / \mathrm{mL})$, the $\mathrm{C} 4$ fraction of complement $(0.59 \mathrm{~g} / \mathrm{L})$ and IgM levels $(4.24 \mathrm{~g} / \mathrm{L})$. Positivity of ANA was found from the immunological routine $(1 / 320$, speckled nuclear pattern) and the absence of anti-ENA antibody, anti-ds DNA antibody, RF, anti-CCP antibody, ACA, ANCA, anti $\beta 2$ glycoprotein anti- 


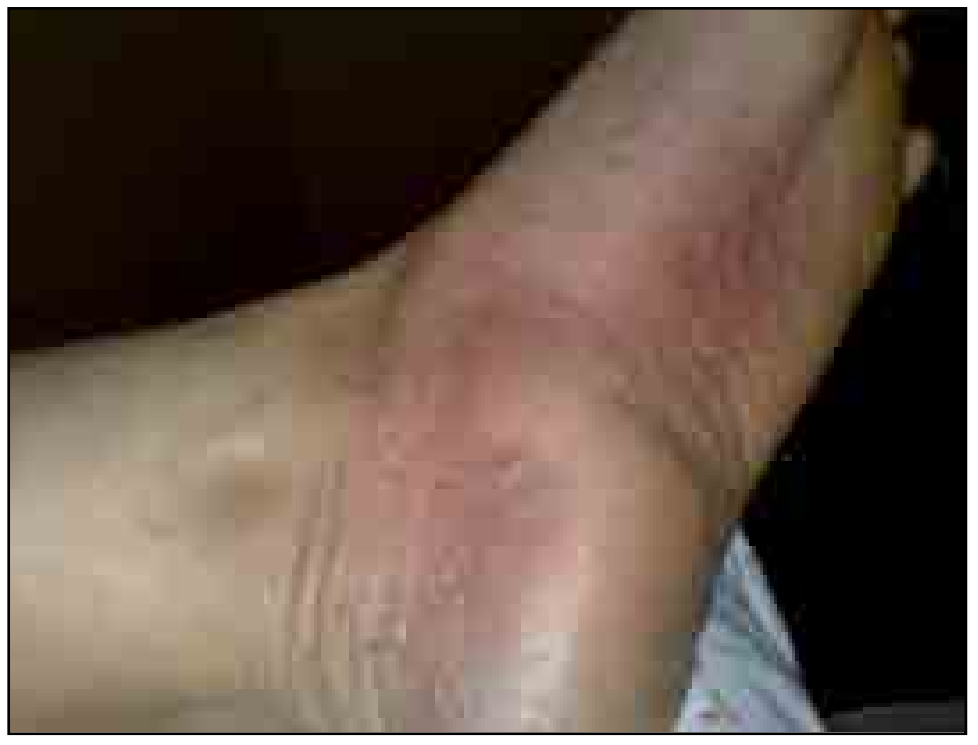

Figure 1 - Erythematous petechial lesions on the ends already in partial evolution phase.

body and cryoglobulins; regular resulted the coagulation and level II examination tests for thrombophilia; finally normal were the chest X-ray, the ultrasonography examination of abdomen, thyroid and salivary glands, as well as the nailfold capillaroscopy. Done the diagnosis of HenochSchonlein purpura, although incomplete, we started therapy with prednisone $25 \mathrm{mg} /$ die, achieving, in about 10 days, a quick

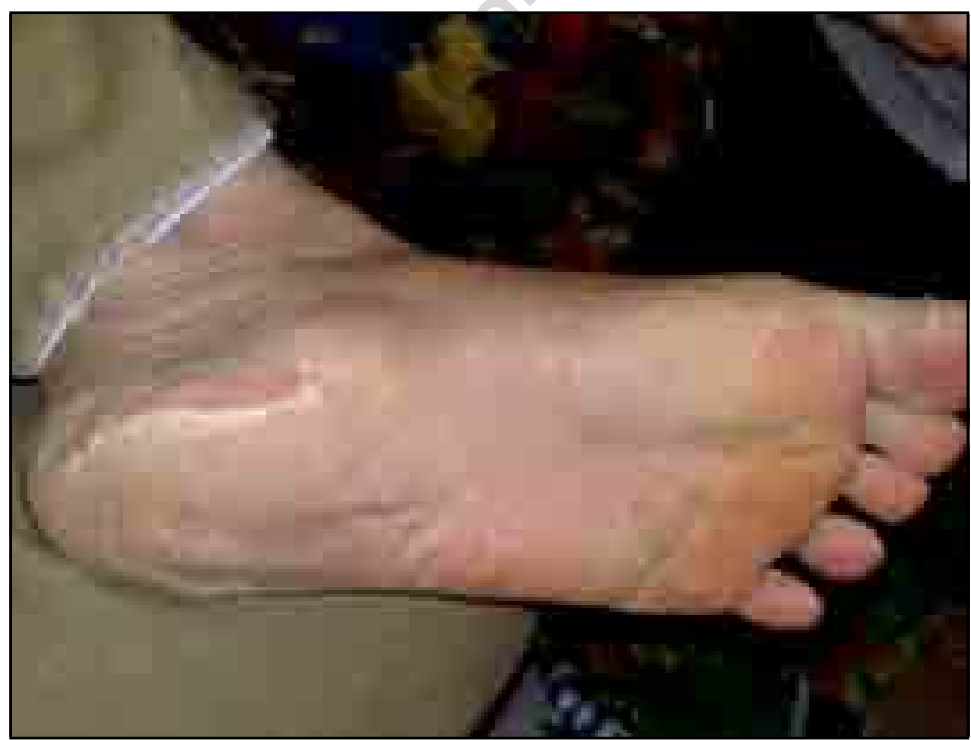

Figure 2 - Erythematous petechial lesions on the upper ends already in partial evolution phase. response and complete resolution of symptoms (Fig. 3).

A few days later, the patient's mother, a 54 years old nurse, came to the same clinic lamenting, for about 36 hours, the appearance of the same symptoms: erythema and purple to the four extremities, fever, arthralgia, mouth ulcers and swelling of the lower lip. Diagnostic investigations showed normality in: blood counts, liver and kidney function tests, serum electrolytes, muscle enzymes, $\mathrm{C} 4$ fraction of complement, immunoglobulins levels and tumor markers; and alterated were the acute phase reactants (ESR $30 \mathrm{~mm}, \mathrm{CRP}$ $19.1 \mathrm{mg} / \mathrm{L})$, the C3 fraction of complement $(0.74 \mathrm{~g} / \mathrm{L})$ and immunoglobulins levels (gamma globulin 22.1\%, IgM 4.24 $\mathrm{g} / \mathrm{L}$ ). The immunological routine revealed the absence of ANA, anti-ENA antibodies, anti-ds DNA antibodies, RF, anti-CCP antibodies, ACA, ANCA, cryoglobulins and anti $\beta 2$ glycoprotein antibodies. The coagulation, level II examination tests for thrombophilia and the thyroid function resulted regular, while positive were the search for anti-thyroid antibodies. ECG, chest X-ray, ultrasonography examination of abdomen and salivary glands, as well as the nailfold capillaroscopy resulted normal, while parenchymal inhomogeneity was detected at the ultrasonography examination of the thyroid. A steroid therapy (deflazacort $30 \mathrm{mg} /$ day) was started for suspected viral triggered vasculitis and for this reason further infectious investigations were initiated. The normality of antistreptolysin title, serodiagnosis of Vidal Wright and anti-hepatitis $C$ serology, the positivity for anti-HBe and total anti- $\mathrm{HBc}$ antibodies, negativity of anti Adenovirus, anti Coxackie virus and anti Echovirus serodiagnosis and the negativity of Adenovirus in the stool was found. Finally the absence of IgM anti-Rubella, CMV, HSV, EBV, Toxoplasma and positivity of IgM and IgG anti parvovirus B19 was detected. The treatment undertaken involved a quick and complete resolution of the symptoms within few days.

The detection of anti Parvovirus B19 antibodies in the mother, suggested a deeper 


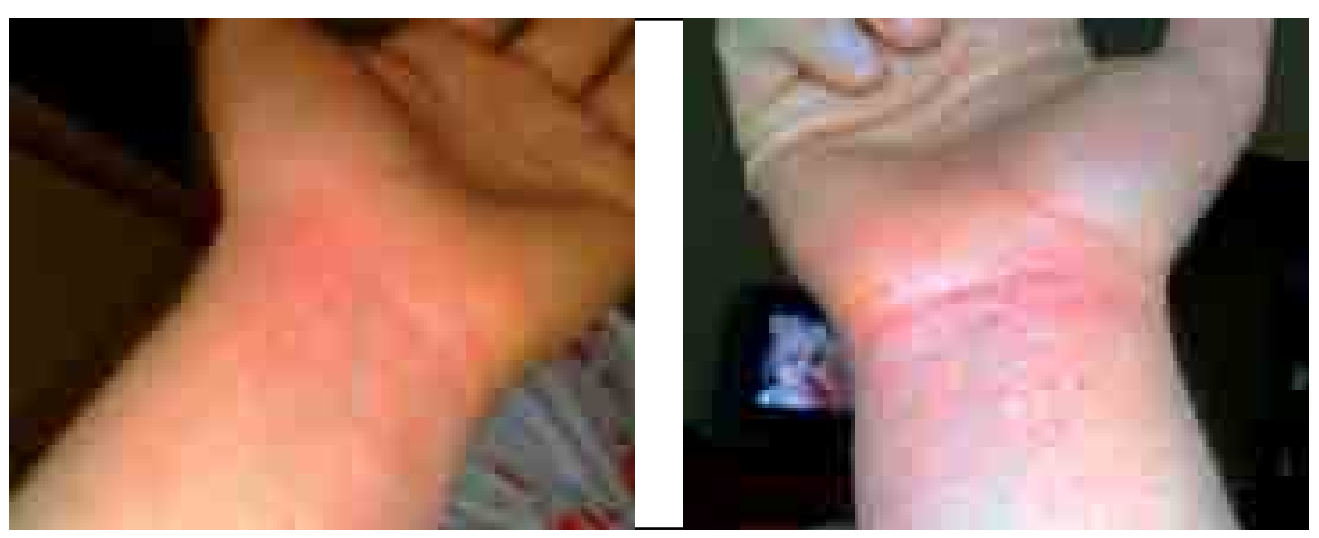

Figure 3 - Healing without sequelae was achieved by the appearance of areas of desquamation in the lesion sites.

infectious investigations also in the daughter, which showed: absence of anti-Rubella, anti CMV, anti HSV, and anti toxoplasma IgM; negativity of EBV and Vidal Wright serodiagnosis; positivity of $\operatorname{IgM}$ and $\operatorname{IgG}$ anti parvovirus B19 research.

At a follow-up visit carried out 3 months after the onset and 2 months after the complete withdrawal of the steroid, a complete remission of symptoms is noted for both patients, together with the normalization of the laboratory tests and the inflammatory markers. Clinical-biohumoral remission was confirmed for 12 months.

\section{DISCUSSION}

The structure of the Parvovirus B 19 includes a capsid formed by two structural proteins with immunogenic activity (VP1 and VP2) and various non-structural proteins as the NS1, decisive for the replication and pathogenicity of the virus. The mechanism by which parvovirus B19 generates lesions is uncertain. The cellular receptor for the virus ( $\mathrm{P}$ antigen) is present on endothelial cells making them susceptible to infection, but not permissive for its replication. The cellular damage may be induced or by a block in the chain reproductive followed by hyperproduction of viral non-structural cytotoxic proteins (NS1), or by a local inflammatory response to a vasculitic-like infection. However, the histology of the lesions is not specific for vasculitis, but it only shows lymphocytic infiltrates in the papillary dermis, associated with blood extravasation. A direct immunofluorescence study showed deposition of IgM and C3 fraction of complement in the papillary dermis vessels wall, suggesting a vascular reaction to antigenic challenge (3). Moreover, the structural protein VP2 of parvovirus B19 was isolated in the endothelium of dermal vessels with immunoistochimical technique (17).

The diagnosis of parvovirus infection is done through the search of the viral DNA in serum by polymerase chain reaction (PCR) or in tissues by in situ hybridization (18). The histological diagnosis, done by means of a light microscopy is based on detection of nuclear eosinophilic inclusions with peripheral chromatid thickening containing viral particles. The antibody response can be assessed by the search for IgM and IgG with ELISA or RIA method; it is demonstrated the presence of specific $\operatorname{IgM}$ up to 6-8 weeks after infection. Serum IgM testing is recommended for the diagnosis of acute infection in immunocompetent patients and the test has demonstrated high sensitivity (89\%) and specificity (99\%) (19). The search for the viral DNA is useful for the diagnosis in patients with transient aplastic crisis or in immunocompromised patients where the antibody response is deficient (20). Anyway PCR does not seem to be more sensitive than IgM antibody assays for the diagnosis of acute parvovirus infection (1). 
The symptomatic variety of PPGS requires differential diagnosis with other rheumatologic and hematologic disease. The differential diagnosis with lymphoproliferative disorders and other thrombocytopenic syndromes is necessary due to the systemic and acute onset, accompanied by hemorrhagic lesions. The onset characterized by fever, abnormalities of the mucosa of the lips and oral cavity, cervical lymphadenopathy, palmar and plantar erythema evolving in desquamation of the extremities, may simulate Kawasaki disease (or mucocutaneous lymphnode syndrome). Other diagnostic suspicion arises to the leukocytoclasic or hypersensitivity vasculitis, characterized by lesions of the small vessels and electively involving the postcapillary venules of the skin; these include the cryoglobulinemic vasculitis and Henoch-Schonlein purpura, the latter characterized by onset with fever and purpuric skin lesions in the perimalleolar region, angina abdominis, transient arthritis and mesangial glomerulonephritis. Among the diseases of rheumatologist interest the differential diagnosis should be made with Still's disease, due to the acute onset and the systemic and cutaneous involvement, although it shows different characteristics (e.g. fleeting rubella-like rash). In most cases, PPGSS requires only symptomatic treatment with nonsteroidal antiinflammatory drugs and antipyretics; the use of corticosteroids is not indicated because it is a self-limiting disease and immunosuppressive therapy may promote the persistence of the virus.

The two clinical cases described above demonstrate that a thorough anamnestic investigation is a fundamental tool in the execution of a correct diagnostic procedure and therefore it can avoids unnecessary laboratory tests and treatment.

\section{REFERENCES}

1. Servey JT, Reamy BV, Hodge J. Clinical Presentations of Parvovirus B19 Infection. Am Fam Physician. 2007; 75: 373-6.

2. Harms M, Feldmann R, Saraut JH. Papularpurpuric "gloves and socks" syndrome. J Amer Acad Derm. 1990; 23: 850-4.
3. Smith PT, Landry ML, Carey H, et al. Papularpurpuric gloves and socks syndrome associated with acute parvovirus B19 infection: case report and review. Clin Infect Dis. 1998; 27: 164-8.

4. Harel L, Straussberb L, Zeharia A, et al. Papular purpuric rash due to Parvovirus B19 with distribution on the distal extremities and the face. Clin Infect Dis. 2002; 35: 1558-61.

5. Yan AC. Parvovirus-associated papular-purpuric "gloves and socks" eruption, with atypical unilateral facial involvement in locus minoris resistentiae. Arch Pediatr Adolesc Med. 2010; 164: 1066.

6. Sklavounou-Andrikopoulou A, Iakovou M, Paikos S, et al. Oral manifestations of papularpurpuric "gloves and socks" syndrome due to parvovirus B19 infection: the first case presented in Greece and review of the literature. Oral Dis. 2004; 10: 118-22.

7. Segura Saint-Gerons R, Ceballos Salobreña A, Gutiérrez Torres P, et al. Papular purpuric gloves and socks syndrome.Presentation of a clinical case. Med Oral Patol Oral Cir Bucal. 2007; 12: E4-6.

8. Carrascosa JM, Bielsa I, Ribera M, Ferrándiz C. Papular-purpuric gloves and socks syndrome related to cytomegalovirus infection. Dermatology. 1995; 191: 269-70.

9. Bagot M, Revuz J. Papular-purpuric "gloves and socks" syndrome: primary infection with parvovirus B19? J Amer Acad Derm. 1991; 25: 341-2.

10. Ruzicka T, Kalka K, Diercks K, Schuppe HC. Papular-purpuric "gloves and socks" syndrome associated with human herpesvirus 6 infection. Arch Derm. 1998; 134: 242-4.

11. Feldmann R, Harms M, Saurat J-H. Papularpurpuric gloves and socks syndrome: not only Parvovirus B19. Dermatology. 1994; 188: 85-7.

12. Gaston PA, Zurowski SM. Arcanobacterium haemolyticum pharyngitis and exanthem. Three case reports and literature review. Arch Derm. 1996; 132: 61-4.

13. van Rooijen MM, Brand CU, Ballmer-Weber BK, et al. Drug-induced papular-purpuric gloves and socks syndrome. Hautarzt 1999; 50: 280-3.

14. Pemira SM, Tolan RW Jr. Mycoplasma pneumoniae infection presenting as bullous papular purpuric gloves and socks syndrome: novel association and review of the literature. Clin Pediatr (Phila). 2011. [Epub ahead of print].

15. Martínez GMJ, Elgueta NA. A family outbreak of parvovirus B19 atypical exanthemas. Report of two cases. Rev Méd Chile. 2008; 136: 620-3.

16. Alfadley A, Aljubran A, Hainau B, Alhokail A. Papular-purpuric "gloves and socks" syndrome in a mother and daughter. J Am Acad Dermatol. 2003; 48: 941-4.

17. Santonja C, Nieto-González G, Santos-Briz 
A, et al. Immunohistochemical detection of Parvovirus B19 in "gloves and socks" papular purpuric syndrome: direct evidence for viral endothelial involvement. report of three cases and review of the literature. Am J Dermatopathol. 2011. [Epub ahead of print].

18. Grilli R, Izquierdo MJ, Fariña MC. Papularpurpuric "gloves and socks" syndrome: polymerase chain reaction demonstration of parvovirus B19 DNA in cutaneous lesions and sera.
J Amer Acad Derm. 1999;41:793-6.

19. Doyle S, Kerr S, O'Keeffe G, et al. Detection of parvovirus B19 IgM by antibody capture enzyme immunoassay: reciver operating characteristic analysis. J Virol Methods 2000; 90: 143-52.

20. Smith-Whitley K, Zhao H, Hodinka RL, et al. Epidemiology of human parvovirus B19 in children with sickle cell disease. Blood. 2004; 103: 422-7. 